\title{
Making the Diffie-Hellman Protocol Identity-Based ${ }^{\star}$
}

\author{
Dario Fiore $^{1 \star \star}$ and Rosario Gennaro ${ }^{2}$ \\ 1 Dipartimento di Matematica ed Informatica - Università di Catania, Italy. fiore@dmi.unict.it \\ ${ }^{2}$ IBM T.J. Watson Research Center - Hawthorne, New York 10532. rosario@us.ibm.com
}

\begin{abstract}
This paper presents a new identity based key agreement protocol. In id-based cryptography (introduced by Adi Shamir in [33]) each party uses its own identity as public key and receives his secret key from a master Key Generation Center, whose public parameters are publicly known.

The novelty of our protocol is that it can be implemented over any cyclic group of prime order, where the Diffie-Hellman problem is supposed to be hard. It does not require the computation of expensive bilinear maps, or additional assumptions such as factoring or RSA.

The protocol is extremely efficient, requiring only twice the amount of bandwith and computation of the unauthenticated basic Diffie-Hellman protocol. The design of our protocol was inspired by MQV (the most efficient authenticated Diffie-Hellman based protocol in the public-key model) and indeed its performance is competitive with respect to MQV (especially when one includes the transmission and verification of certificates in the MQV protocol, which are not required in an id-based scheme). Our protocol requires a single round of communication in which each party sends only 2 group elements: a very short message, especially when the protocol is implemented over elliptic curves.

We provide a full proof of security in the Canetti-Krawczyk security model for key exchange, including a proof that our protocol satisfies additional security properties such as forward secrecy, and resistance to reflection and key-compromise impersonation attacks.
\end{abstract}

\section{Introduction}

Identity-based cryptography was introduced in 1984 by Adi Shamir [33]. The goal was to simplify the management of public keys and in particular the association of a public key to the identity of its holder. Usually such binding of a public key to an identity is achieved by means of certificates which are signed statements by trusted third parties that a given public key belongs to a user. This requires users to obtain and verify certificates whenever they want to use a specific public key, and the management of public key certificates remains a technically challenging problem.

Shamir's idea was to allow parties to use their identities as public keys. An id-based scheme works as follows. A trusted Key Generation Center (KGC) generates a master public/secret key pair, which is known to all the users. A user with identity $I D$ receives from the KGC a secret key $S_{I D}$ which is a function of the string $I D$ and the KGC's secret key (one can think of $S_{I D}$ as a signature by the KGC on the string $I D$ ). Using $S_{I D}$ the user can then perform cryptographic tasks. For example in the case of $i d$-based encryption any party can send an encrypted message to the user with identity $I D$ using the string $I D$ as a public key and the user (and only the user and the KGC) will be able to decrypt it using $S_{I D}$. Note that the sender can do this even if the recipient has not obtained yet his secret key from the KGC. All the sender needs to know is the recipient's identity and the public parameters of the KGC. This is the major advantage of id-based encryption.

Id-Based Key Agreement and its Motivations. This paper is concerned with the task of $i d$ based key agreement. Here two parties Alice and Bob, with identities $A, B$ and secret keys $S_{A}, S_{B}$

\footnotetext{
* An extended abstract of this paper appears in the proceedings of CT-RSA 2010

${ }^{\star \star}$ Work done while visiting NYU and IBM Research.
} 
respectively, want to agree on a common shared key, in an authenticated manner (i.e. Alice must be sure that once the key is established, only Bob knows it - and viceversa). Since key agreement is inherently an interactive protocol (both parties are "live" and ready to establish a session) there is a smaller gain in using an id-based solution: indeed certificates and public keys can be easily sent as part of the protocol communication.

Yet the ability to avoid sending and verifying public key certificates is a significant practical advantage (see e.g. [36]). Indeed known shortcomings of the public key setting are the requirement of centralized certification authorities, the need for parties to cross-certify each other (via possibly long certificate chains), and the management of some form of large-scale coordination and communication (possibly on-line) to propagate certificate revocation information. Identity-based schemes significantly simplify identity management by bypassing the certification issues. All a party needs to know in order to generate a shared key is its own secret key, the public information of the KGC, and the identity of the communication peer (clearly, the need to know the peer's identity exists in any scheme including a certificate-based one).

Another advantage of identity-based systems is the versatility with which identities may be chosen. Since identities can be arbitrary string, they can be selected according to the function and attributes of the parties (rather than its actual "name"). For example in vehicular networks a party may be identified by its location ("the checkpoint at the intersection of a and b") or in military applications a party can be identified by its role ("platoon x commander"). This allows parties to communicate securely with the intended recipient even without knowing its "true" identity but simply by the definition of its function in the network.

Finally, identities can also include additional attributes which are temporal in nature: in particular an "expiration date" for an identity makes revocation of the corresponding secret key much easier to achieve.

For the reasons described above, id-based KA protocols are very useful in many systems where bandwith and computation are at a premium (e.g. sensor networks), and also in ad-hoc networks where large scale coordination is undesirable, if not outright impossible. Therefore it is an important question to come up with very efficient and secure id-based KA protocols.

Previous work on iD-BASed Key agreement. Following Shamir's proposal of the concept of id-based cryptography, some early proposals for id-based key agreement appeared in the literature: we refer in particular to the works of Okamoto [28] (later improved in [29]) and Gunther [21]. A new impetus to this research area came with the breakthrough discovery of bilinear maps and their application to id-based encryption in [5]: starting with the work of Sakai et al. [32] a large number of id-based KA protocols were designed that use pairings as tool. We refer the readers to [6] and [12] for surveys of these pairing-based protocols.

The main problem with the current state of the art is that many of these protocols lack a proof of security, and some have even been broken. Indeed only a few (e.g., [8,37]) have been proven according to a formal definition of security.

Our Contribution. By looking at prior work we see that provably secure id-based KAs require either groups that admit bilinear maps [8,37], or to work over a composite RSA modulus [29].

This motivated us to ask the following question: can we find an efficient and provably secure id-based KA protocol such that:

1. it that can be implemented over any cyclic group in which the Diffie-Hellman problem is supposed to be hard. The advantages of such a KA protocol would be several, in particular: (i) it 
would avoid the use of computationally expensive pairing computations; (ii) it could be implemented over much smaller groups (since we could use 'regular' elliptic curves, rather than the ones that admit efficient pairings computations for high security levels, or the group $Z_{N}^{*}$ for a composite $N$ needed for Okamoto-Tanaka).

2. it is more efficient than any KA protocols in the public key model (such as MQV [26]), when one includes the transmission and verification of certificates which are not required in an id-based scheme. This is a very important point since, as we pointed out earlier in this Section, id-based KA protocols are only relevant if they outperform PKI based ones in efficiency.

Our new protocol presented in this paper (whose description appears in Figure 1), achieves all these features.

\section{The IB-KA Protocol}

Setting: A Key Generation Center (KGC) chooses a group $\mathbb{G}$ of prime order $q$ together with a random generator $g \in \mathbb{G}$ and an exponent $x \stackrel{\$}{\leftarrow} \mathbb{Z}_{q}$. KGC publishes $\mathbb{G}, q, g, y=g^{x}$ and two hash functions $H_{1}, H_{2}$.

Key Derivation: A user with identity $U$ receives its private key $\left(r_{U}, s_{U}\right)$ from the KGC computed as the Schnorr's signature of the string $U$ under public key $y$. That is $r_{U}=g^{k_{U}}$ for $k_{U} \stackrel{\$}{\leftarrow} \mathbb{Z}_{q}$ and $s_{U}=k_{U}+x H_{1}\left(U, r_{U}\right) \bmod q$.

Key agreement: $A$ and $B$ choose ephemeral private exponents $t_{A}$ and $t_{B}$, respectively.

$$
\begin{aligned}
& A \longrightarrow r_{A}, u_{A}=g^{t_{A}} \longrightarrow B \\
& 4, r_{B}, u_{B}=g^{t_{B}} \\
& z_{1}=\left(u_{B} r_{B} y^{H_{1}\left(B, r_{B}\right)}\right)^{t_{A}+s_{A}} \quad z_{1}=\left(u_{A} r_{A} y^{H_{1}\left(A, r_{A}\right)}\right)^{t_{B}+s_{B}} \\
& z_{2}=u_{B}^{t_{A}} \quad z_{2}=u_{A}^{t_{B}} \\
& Z=H_{2}\left(z_{1}, z_{2}\right)
\end{aligned}
$$

Fig. 1. $A$ and $B$ share session key $Z$. See Section 3 for more specific details.

It can be implemented over any cyclic group over which the Diffie-Hellman problem is assumed to be hard. In addition it requires an amount of bandwith and computation similar to the unauthenticated basic Diffie-Hellman protocol. Indeed our new protocol requires a single round of communication in which each party sends just two group elements (as opposed to one in the DiffieHellman protocol). Each party must compute four exponentiations to compute the session key (as opposed to two in the Diffie-Hellman protocol).

A similar favorable comparison holds with the Okamoto-Tanaka protocol in [29]. While that protocol requires only two exponentiations, it does works over $Z_{N}^{*}$ therefore requiring the use of a larger group size, which almost totally absorbs the computational advantage, and immediately implies a much larger bandwith requirement. Detailed efficiency comparisons to other protocols in the literature are discussed in Section 6. 
We present a full proof of security of our protocol in the Canetti-Krawczyk security model. Our results hold in the random oracle model, under the Strong Diffie-Hellman Assumption. We also present some variations of our protocol that can be proven secure under the basic Computational Diffie-Hellman Assumption. Our protocol can be proven to satisfy additional desirable security properties such as perfect forward secrecy ${ }^{3}$, and resistance to reflection and key-compromise impersonation attacks.

Our Approach. The first direction we took in our approach was to attempt to analyze the idbased KA protocols by Gunther [21] and Saeednia [31]. They also work over any cyclic group where the Diffie-Hellman problem is assumed to be hard, but lack a formal proof of security. While the original protocols cannot be shown to be secure, we were able to prove the security of modified versions of them. Nevertheless these two protocols were not very satisfactory solutions for the problem we had set out to solve, particularly for reasons of efficiency since they required a large number of exponentiations, which made them less efficient than say MQV with certificates. The security analysis of these modified Gunther and Saeednia protocols will be included in the final version.

Our protocol improves over these two protocols by using Schnorr's signatures [34], rather than ElGamal, to issue secret keys to the users. The simpler structure of Schnorr's signatures permits a much more efficient computation of the session key, resulting in less exponentiations and a single round protocol. Our approach was inspired by the way the MQV protocol [26] achieves implicit authentication of the session key. Indeed our protocol can be seen as an id-based version of the MQV protocol.

\section{Preliminaries}

In this section we present some standard definitions needed in the rest of the paper.

Let $\mathbb{N}$ the set of natural numbers. We will denote with $\ell \in \mathbb{N}$ the security parameter. The partecipants to our protocols are modeled as probabilistic Turing machines whose running time is bounded by some polynomial in $\ell$. If $S$ is a set, we denote with $s \stackrel{\$}{\leftarrow} S$ the process of selecting an element uniformly at random from $S$.

Definition 1 (Negligible function). A function $\epsilon(\ell)$ is said to be negligible if for every polynomial $p(\ell)$ there exists a positive integer $c \in \mathbb{N}$ such that $\forall \ell>c$ we have $\epsilon(\ell)<1 / p(\ell)$.

In the following assume $\mathbb{G}$ to be a cyclic multiplicative group of order $q$ where $q$ is a $\ell$-bit long prime. We assume that there are efficient algorithms to perform multiplication and membership test in $\mathbb{G}$. Finally we denote with $g$ a generator of $\mathbb{G}$.

Assumption 1 (Computational Diffie-Hellman [16]) We say that the Computational DiffieHellman (CDH) Assumption (for $\mathbb{G}$ and $g$ ) holds if for any probabilistic polynomial time adversary $\mathcal{A}$ the probability that $\mathcal{A}$ on input $\left(\mathbb{G}, g, g^{u}, g^{v}\right)$ outputs $W$ such that $W=g^{u v}$ is negligible in $\ell$. The probability of success of $\mathcal{A}$ is taken over the uniform random choice of $u, v \in \mathbb{Z}_{q}$ and the coin tosses of $\mathcal{A}$.

\footnotetext{
${ }^{3}$ We can prove PFS only in the case the adversary was passive in the session that he is attacking - though he can be active in other sessions. As proven by Krawczyk in [25], this is the best that can be achieved for 1-round protocols with implicit authentication, such as ours.
} 
The CDH Assumption has a Decisional version in which no adversary can actually recognize the value $g^{u v}$ when given $g^{u}, g^{v}$. In the proof of our basic protocol we are going to need the ability to perform such decisions when one of the two elements is fixed, while still assuming that the CDH holds. The assumption below basically says that the CDH Assumption still holds in the presence of an oracle $\mathrm{DH}(U, \cdot, \cdot)$ that solves the decisional problem ${ }^{4}$ for a fixed $U$.

Assumption 2 (Strong-DH Assumption [1]) We say that the Strong-DH (SDH) Assumption holds (for $\mathbb{G}$ and $g$ ) if the CDH Assumption holds even in the presence of an oracle $\mathrm{DH}(U, \cdot, \cdot)$ that on input two elements $\hat{V}, \hat{W}$ in the group generated by $g$, output "yes" if and only if $\hat{W}$ is the Diffie-Hellman of $U$ and $\hat{V}$.

The oracle DH for the Decisional DH problem exists for some groups $\mathbb{G}$, e.g. the ones that admit a bilinear map. We stress, however that we need the oracle only for the proof of security, and it is not needed in the execution of the protocol by the real-life parties. This means that we can efficiently implement our protocol over any cyclic group $\mathbb{G}$.

The oracle DH for the Decisional DH problem exists for some groups $\mathbb{G}$, e.g. the ones that admit a bilinear map. We stress, however that we need the oracle only for the proof of security, and it is not needed in the execution of the protocol by the real-life parties. This means that we can efficiently implement our protocol over any cyclic group $\mathbb{G}$.

The question, then, is the real-life meaning of a proof under the Strong-DH assumption when the protocol is implemented over a group $\mathbb{G}$ that does not admit such oracle $\mathrm{DH}$. If we prove the security of our protocol under the SDH assumption, then if a successful adversary can be constructed one of two things must be true:

1. either the CDH Assumption is false

2. or we have a proof that the hardness of the Decisional problem is implied by the CDH Assumption (in other words the $\mathrm{CDH}$ and $\mathrm{DDH}$ Assumptions are equivalent). Indeed in this case the $\mathrm{CDH}$ holds, and the protocol is insecure, which means that the oracle $\mathrm{DH}$ cannot exists (if it existed, given that the $\mathrm{CDH}$ holds, the protocol should be secure).

In other words, while proofs under the Strong-DH assumption do not necessarily offer a constructive cryptanalysis of a conjectured hard problem in case of a successful attack, they do offer the "dual" ability to prove the equivalence of the CDH Assumption (with any other additional assumption required by the proof) with the DDH Assumption over the underlying group.

\subsection{Definitions for identity-based key agreement}

The security of our protocols is analyzed in a version of the Canetti-Krawczyk (CK) $[9,10]$ model for key agreement, adapted to the identity-based setting. We present an informal summary of the model and we refer the reader to $[9,10]$ for details.

An identity-based key-agreement protocol is runned by parties interacting in a network where each party is identified by a unique identity which is publicly known to all the other parties (e.g. Alice's identity is a string $I D_{A}$ ). In addition there exists a trusted entity called Key Generation Center (KGC) that generates the public parameters of the system and also issues secret keys to

\footnotetext{
${ }^{4}$ We remark that in recent papers the name strong Diffie-Hellman assumption was used to denote a different conjecture defined over bilinear groups [4]. In this paper, we refer to the original terminology from [1]
} 
users associated with their public identities, e.g. the KGC generates a secret key $S K_{A}$ associated to $I D_{A}$.

An instance of the protocol is called a session. The two parties participating in the session are called its peers. Each peer maintains a session state which contains incoming and outgoing messages and its random coins. If the session is completed then each party outputs a session key and erases its session state. A session may also be aborted. In this case no session key is generated.

Each party assigns an unique identifier to a session he is participating in. For simplicity, we assume it to be the quadruple (Alice, Bob, $\left.m_{O u t}, m_{I n}\right)$ where Alice is the identity of the party, Bob its peer, $m_{O u t}$ and $m_{I n}$ are the outgoing and incoming messages, respectively, for Alice. If Alice holds a session $\left(\right.$ Alice, Bob, $\left.m_{\text {Out }}, m_{I n}\right)$ and Bob holds a session $\left(B o b\right.$, Alice, $\left.m_{\text {In }}, m_{\text {Out }}\right)$ then the two sessions are matching.

The adversary The CK definition models a very realistic adversary which basically controls all communication in the network. In particular it can intercept and modify messages exchanged by parties, delay or block their delivery, inject its own messages, schedule sessions etc. The adversary is allowed to choose the identities of the parties, and obtain private keys from the KGC for identities of its choice.

Finally we allow the adversary to access some of the parties' secret information, via the following attacks: party corruption, state-reveal queries and session-key queries. When an adversary corrupts a party, it learns its private information (the private key and all session states and session keys currently stored), and it later controls its actions. In a state-reveal query to a party running a session, the adversary learns the session state for that session (since we assume that session states are erased at the end of the session, such query makes sense only against sessions that are still incomplete). Finally a session-key query allows the adversary to learn the session key of a complete session. A session is called exposed if it or its matching session (if existing) is compromised by one of the attacks above.

Security Definition Let $\mathcal{A}$ be a probabilistic polynomial time adversary modeled as described above. Then consider the following experiment running $\mathcal{A}$.

At the beginning of the game the adversary receives in input the public parameters of the system (generated by the KGC) and then can perform all the actions described in the section before.

At some point, $\mathcal{A}$ chooses a test session among all the completed and unexposed sessions. We toss a random bit $b \stackrel{\$}{\leftarrow}\{0,1\}$. If $b=0$ we give $\mathcal{A}$ the session key $K_{0}$ of the test session. Otherwise we take a random session key $K_{1}$ and provide $\mathcal{A}$ with $K_{1}$.

After having received $K_{b}$, the adversary can continue to perform its actions against the protocol with the exception that it cannot expose the test session. At the end of the game $\mathcal{A}$ outputs a bit $b^{\prime}$ as its guess for $b$.

Definition 2. An identity-based key-agreement protocol is said to be secure if for any PPT adversary $\mathcal{A}$ the following holds:

1. if two uncorrupted parties complete matching sessions then they output the same session key with overwhelming probability;

2. the probability that $\mathcal{A}$ guesses the correct $b$ in the above experiment is at most $1 / 2$ plus a negligible fraction of the security parameter.

We define the advantage of $\mathcal{A}$ as $\mathbf{A d v}_{\mathcal{A}}^{I B-K A}=\left|\operatorname{Pr}\left[b=b^{\prime}\right]-1 / 2\right|$. 


\section{The New Protocol IB-KA}

Protocol setup. The Key Generation Center (KGC) chooses a group $\mathbb{G}$ of prime order $q$ (where $q$ is $\ell$-bits long), a random generator $g \in \mathbb{G}$ and two hash functions $H_{1}:\{0,1\}^{*} \rightarrow \mathbb{Z}_{q}$ and $H_{2}: \mathbb{Z}_{q} \times \mathbb{Z}_{q} \rightarrow\{0,1\}^{\ell}$. Then it picks a random $x \stackrel{\$}{\leftarrow} \mathbb{Z}_{q}$ and sets $y=g^{x}$. Finally the KGC outputs the public parameters $M P K=\left(\mathbb{G}, g, y, H_{1}, H_{2}\right)$ and keeps the master secret key $M S K=x$ for itself.

Key Derivation. A user with identity ID receives, as its secret key, a Schnorr's signature [34] of the message $m=I D$ under public key $y$. More specifically, the KGC after verifying the user's identity, creates the associated secret key as follows. First it picks a random $k \stackrel{\$}{\leftarrow} \mathbb{Z}_{q}$ and sets $r_{I D}=g^{k}$. Then it uses the master secret key $x$ to compute $s_{I D}=k+H_{1}\left(I D, r_{I D}\right) x .\left(r_{I D}, s_{I D}\right)$ is the secret key returned to the user. The user can verify the correctness of its secret key by using the public key $y$ and checking the equation $g^{s_{I D}} \stackrel{?}{=} r_{I D} \cdot y^{H_{1}\left(I D, r_{I D}\right)}$.

A protocol session. Let's assume that Alice wants to establish a session key with Bob. Alice owns secret key $\left(r_{A}, s_{A}\right)$ and identity $A$ while Bob has secret key $\left(r_{B}, s_{B}\right)$ and identity $B$.

Alice selects a random $t_{A} \stackrel{\$}{\leftarrow} \mathbb{Z}_{q}$, computes $u_{A}=g^{t_{A}}$ and sends the message $\left\langle A, r_{A}, u_{A}\right\rangle$ to Bob. Analogously Bob picks a random $t_{B} \stackrel{\$}{\leftarrow} \mathbb{Z}_{q}$, computes $u_{B}=g^{t_{B}}$ and sends $\left\langle B, r_{B}, u_{B}\right\rangle$ to Alice. After the parties have exchanged these two messages, they are able to compute the same session key $Z=H_{2}\left(z_{1}, z_{2}\right)$. In particular Alice computes

$$
z_{1}=\left(u_{B} r_{B} y^{H_{1}\left(B, r_{B}\right)}\right)^{t_{A}+s_{A}} \text { and } z_{2}=u_{B}^{t_{A}} .
$$

On the other hand Bob computes

$$
z_{1}=\left(u_{A} r_{A} y^{H_{1}\left(A, r_{A}\right)}\right)^{t_{B}+s_{B}} \text { and } z_{2}=u_{A}^{t_{B}} .
$$

It is easy to see that both the parties are computing the same values $z_{1}=g^{\left(t_{A}+s_{A}\right)\left(t_{B}+s_{B}\right)}$ and $z_{2}=g^{t_{A} t_{B}}$. The state of a user $I D$ during a protocol session contains only the fresh random exponent $t_{I D}$. We assume that after a session is completed, the parties erase their state and keep only the session key.

Remark: In the next section we show that protocol IB-KA is secure under the Strong Diffie-Hellman Assumption. However, in Section 5 we show how to modify IB-KA to obtain security under the basic $\mathrm{CDH}$ Assumption, at the cost of a slight degradation in efficiency.

\section{Security Proof}

We prove the security of the protocol by a usual reduction argument. More precisely we show how to reduce the existence of an adversary breaking the protocol into an algorithm that is able to break the SDH Assumption with non-negligible probability. The adversary is modeled as a CK attacker: (see Section 2.1 for details): in particular it will choose a test session among the complete and unexposed sessions and will try to distinguish between its real session key and a random one.

In our reduction we will make use of the General Forking Lemma, stated by Bellare and Neven in [2]. It follows the original forking lemma of Pointcheval and Stern [30], but, unlike that, it makes no mention of signature schemes and random oracles. In this sense it is more general and it can be used to prove the security of our protocol. We briefly recall it in the following. 
Lemma 1 (General Forking Lemma [2]). Fix an integer $Q \geq 1$ and a set $H$ of size $|H| \geq 2$. Let $\mathcal{B}$ be a randomized algorithm that on input $x, h_{1}, \ldots, h_{Q}$ returns a pair $(J, \sigma)$ where $J \in\{0, \ldots, Q\}$ and $\sigma$ is referred as side output. Let $I G$ be a randomized algorithm called the input generator. Let $\operatorname{acc}_{\mathcal{B}}=\operatorname{Pr}\left[J \geq 1: x \stackrel{\$}{\leftarrow} I G, h_{1}, \ldots, h_{Q} \stackrel{\$}{\leftarrow} H ;(J, \sigma) \stackrel{\$}{\leftarrow} \mathcal{B}\left(x, h_{1}, \ldots, h_{Q}\right)\right]$ be the accepting probability of $\mathcal{B}$.

The forking algorithm $F_{\mathcal{B}}$ associated to $\mathcal{B}$ is the randomized algorithm that takes in input $x$ and proceeds as follows:

Algorithm $F_{\mathcal{B}}(x)$

Pick random coins $\rho$ for $\mathcal{B}$

$h_{1}, \ldots, h_{Q} \stackrel{\$}{\leftarrow} H$

$(J, \sigma) \stackrel{\$}{\leftarrow} \mathcal{B}\left(x, h_{1}, \ldots, h_{Q} ; \rho\right)$

If $J=0$ then return $(0, \perp, \perp)$

$h_{1}^{\prime}, \ldots, h_{Q}^{\prime} \stackrel{\$}{\leftarrow} H$

$\left(J^{\prime}, \sigma^{\prime}\right) \stackrel{\$}{\leftarrow} \mathcal{B}\left(x, h_{1}, \ldots, h_{J-1}, h_{J}^{\prime}, \ldots, h_{Q}^{\prime}, ; \rho\right)$

If $\left(J=J^{\prime}\right.$ and $\left.h_{J} \neq h_{J}^{\prime}\right)$ then return $\left(1, \sigma, \sigma^{\prime}\right)$

Else return $(0, \perp, \perp)$.

Let $f r k=\operatorname{Pr}\left[b=1: x \stackrel{\$}{\leftarrow} I G ;\left(b, \sigma, \sigma^{\prime}\right) \stackrel{\$}{\leftarrow} F_{\mathcal{B}}(x)\right]$. Then frk $\geq a c c_{\mathcal{B}}\left(\frac{a c c \mathcal{B}}{Q}-\frac{1}{|H|}\right)$.

Roughly speaking the lemma says that if an algorithm $\mathcal{B}$ accepts with some non-negligible probability, then a "rewind" of $\mathcal{B}$ is likely to accept roughly with the same probability (more specifically the probability squared). If we look at the details of this lemma, the intuitions are that: (1) $h_{1}, \ldots, h_{Q}$ can be seen as the set of replies to random oracle queries made by the original adversary and (2) the forking algorithm implements the rewinding. Moreover it is important that in $F_{\mathcal{B}}$ the two executions of $\mathcal{B}$ are run with the same random coins. We defer to [2] for the proof of the lemma.

Theorem 3. Under the Strong-DH Assumption, if we model $\mathrm{H}_{1}$ and $\mathrm{H}_{2}$ as random oracles, then protocol IB-KA is a secure identity-based key agreement protocol.

Proof. For sake of contradiction let us suppose there exists a PPT adversary $\mathcal{A}$ that has nonnegligible advantage $\epsilon$ into breaking the protocol IB-KA, then we show how to build a solver algorithm $S$ for the CDH problem.

In our reduction we will proceed into two steps. First, we describe an intermediate algorithm $\mathcal{B}$ (i.e. the simulator) that interacts with the IB-KA adversary $\mathcal{A}$ and returns a side output $\sigma$. Second, we will show how to build an algorithm $S$ that exploits $F_{\mathcal{B}}$, the forking algorithm associated with $\mathcal{B}$, to solve the $\mathrm{CDH}$ problem under the Strong-DH Assumption.

$\mathcal{B}$ receives in input a tuple $(\mathbb{G}, g, U, V)$, where $U=g^{u}, V=g^{v}$ and $u, v$ are random exponents in $\mathbb{Z}_{q}$, and a set of random elements $h_{1}, \ldots, h_{Q} \in \mathbb{Z}_{q}$. The simulator is also given access to a DH oracle $\mathrm{DH}(U, \cdot, \cdot)$ that on input $(\hat{V}, \hat{W})$ answers "yes" if $(U, \hat{V}, \hat{W})$ is a valid DDH tuple. The side output of $\mathcal{B}$ is $\sigma \in \mathbb{G} \times \mathbb{Z}_{q}$ or $\perp$. Let $n$ be an upper bound to the number of sessions of the protocol run by the adversary $\mathcal{A}$ and $Q_{1}$ and $Q_{2}$ be the number of queries made by $\mathcal{A}$ to the random oracles $H_{1}, H_{2}$ respectively. Moreover, let $Q_{c}$ be the number of users corrupted by $\mathcal{A}$ and $Q=Q_{1}+Q_{c}+1$.

$$
\text { Algorithm } \mathcal{B}^{\operatorname{DH}(U, \cdot, \cdot)}\left(\mathbb{G}, g, U, V, h_{1}, \ldots, h_{Q}\right)
$$


Initialize $c t r \leftarrow 0 ;$ bad $\leftarrow$ false; empty tables $\overline{H_{1}}, \overline{H_{2}}$;

Run $\mathcal{A}$ on input $(\mathbb{G}, g, y=U)$ as the public parameters of the protocol and simulates the protocol's environment for $\mathcal{A}$ as follows:

Guess the test session by choosing at random the user (let us call him Bob) and the order number of the test session. If $n$ is an upper bound to the number of all the sessions initiated by $\mathcal{A}$ then the guess is right with probability at least $1 / n$.

$H_{2}$ queries On input a pair $\left(z_{1}, z_{2}\right)$ :

If $\overline{H_{2}}\left[z_{1}, z_{2}\right]=\perp$ : choose a random string $Z \in\{0,1\}^{\ell}$ and store $\overline{H_{2}}\left[z_{1}, z_{2}\right]=Z$

Return $\overline{H_{2}}\left[z_{1}, z_{2}\right]$ to $\mathcal{A}$

$H_{1}$ queries On input $(I D, r)$ :

If $\overline{H_{1}}[I D, r]=\perp$, then $c t r \leftarrow c t r+1 ; \overline{H_{1}}[I D, r]=h_{c t r}$

Return $\overline{H_{1}}[I D, r]$ to $\mathcal{A}$

Party Corruption When $\mathcal{A}$ asks to corrupt party $I D \neq B$, then:

$c t r \leftarrow c t r+1 ; s \stackrel{\$}{\leftarrow} \mathbb{Z}_{q} ; r=g^{s} y^{-h_{c t r}}$

If $\overline{H_{1}}[I D, r] \neq \perp$ then bad $\leftarrow$ true

Store $\overline{H_{1}}[I D, r]=h_{c t r}$ and return $(r, s)$ as $I D$ 's private key.

For the case of Bob, the simulator simply chooses the $r_{B}$ component of Bob's private key by picking a random $k_{B} \stackrel{\$}{\leftarrow} \mathbb{Z}_{q}$ and setting $r_{B}=g^{k_{B}}$. We observe that in this case $\mathcal{B}$ is not able to compute the corresponding $s_{B}$. However, since Bob is the user guessed for the test session, we can assume that the adversary will not ask for his secret key.

Simulating sessions First we describe how to simulate sessions different from the test session. Here the main point is that the adversary is allowed to ask session-key queries and thus the simulator must be able to produce the correct session key for each of these sessions. The simulator has full information about all the users' secret keys except Bob. Therefore $\mathcal{B}$ can easily simulate all the protocol sessions that do not include Bob, and answer any of the attacker's queries about these sessions. Hence we concentrate on describing how $\mathcal{B}$ simulates interactions with Bob.

Assume that Bob has a session with Charlie (whose identity is the string $C$ ). If Charlie is an uncorrupted party this means that $\mathcal{B}$ will generate the messages on behalf of him. In this case $\mathcal{B}$ knows Charlie's secret key and also has chosen his ephemeral exponent $t_{C}$. Thus it is trivial to see that $\mathcal{B}$ has enough information to compute the correct session key. The case when the adversary presents a message $\left\langle C, r_{C}, u_{C}\right\rangle$ to Bob as coming from Charlie is more complicated. Here is where $\mathcal{B}$ makes use of the oracle $\operatorname{DH}(y, \cdot, \cdot)$ to answer a session-key query about this session. The simulator replies with a message $\left\langle B, r_{B}, u_{B}=g^{t_{B}}\right\rangle$ where $t_{B}$ is chosen by $\mathcal{B}$. Recall that the session key is $H_{2}\left(z_{1}, z_{2}\right)$ with $z_{1}=g^{\left(s_{C}+t_{C}\right)\left(s_{B}+t_{B}\right)}$ and $z_{2}=u_{C}^{t_{B}}$. So $z_{1}$ is the Diffie-Hellman result of the values $u_{C} g^{s_{C}}$ and $u_{B} g^{s_{B}}$, where $g^{s_{C}}=r_{C} y^{H_{1}\left(C, r_{C}\right)}$ and $g^{s_{B}}=r_{B} y^{H_{1}\left(B, r_{B}\right)}$ can be computed by the simulator. Notice also that the simulator knows $t_{B}$ and $k_{B}$ (the discrete log of $r_{B}$ in base $g)$. Therefore it checks if $\overline{H_{2}}\left[z_{1}, z_{2}\right]=Z$ where $z_{2}=u_{C}^{t_{B}}$ and $\mathrm{DH}\left(y, u_{C} g^{s_{C}}, \overline{z_{1}}\right)=$ "yes" where $\overline{z_{1}}=\frac{z_{1}}{\left(u_{C} g^{s} C\right)^{\left(k_{B}+t_{B}\right) H_{1}\left(B, r_{B}\right)^{-1}}}$. If $\mathcal{B}$ finds a match then it outputs the corresponding $Z$ as session key for Bob. Otherwise it generates a random $\zeta \stackrel{\$}{\leftarrow}\{0,1\}^{\ell}$ and gives it as response to the adversary. Later, for each query $\left(z_{1}, z_{2}\right)$ to $H_{2}$, if $\left(z_{1}, z_{2}\right)$ satisfies the equation above it sets $\overline{H_{2}}\left[z_{1}, z_{2}\right]=\zeta$ and answers with $\zeta$. This makes oracle's answers consistent. 
In addition observe that the simulator can easily answer to state reveal queries as it chooses the fresh exponents on its own.

Simulating the test session Let $\left\langle B, \rho_{B}, u_{B}=g^{t_{B}}\right\rangle$ be the message from Bob to Alice sent in the test session. We notice that such message may be sent by the adversary who is trying to impersonate Bob. In this case $\mathcal{A}$ may use a value $\rho_{B}=g^{\lambda_{B}}$ of its choice as the public component of Bob's private key (i.e. different than $r_{B}=g^{k_{B}}$ which $\mathcal{B}$ simulated and for which it knows $\left.k_{B}\right) . \mathcal{B}$ responds with the message $\left\langle A, r_{A}, u_{A}=V\right\rangle$ as coming from Alice. Finally $\mathcal{B}$ provides $\mathcal{A}$ with a random session key.

Run until $\mathcal{A}$ halts and outputs its decision bit

If $\overline{H_{1}}\left[B, \rho_{B}\right]=\perp$ then set $c t r \leftarrow c t r+1$ and $\overline{H_{1}}\left[B, \rho_{B}\right]=h_{c t r}$

If $b a d=$ true then return $(0, \perp)$

Let $i \in\{1, \ldots, Q\}$ such that $H_{1}\left(B, \rho_{B}\right)=h_{i}$

Let $Z=H_{2}\left(z_{1}, z_{2}\right)$ be the correct session key for the test session where

$z_{1}=\left(u_{A} r_{A} y^{H_{1}\left(A, r_{A}\right)}\right)^{\left(t_{B}+\lambda_{B}+x h_{i}\right)}$ and $z_{2}=u_{A}^{t_{B}}$.

If $\mathcal{A}$ has success into distinguishing $Z$ from a random value it must necessarily query the correct pair $\left(z_{1}, z_{2}\right)$ to the random oracle $H_{2}$. This means that $\mathcal{B}$ can efficiently find the pair $\left(z_{1}, z_{2}\right)$ in the table $\overline{H_{2}}$ using the Strong-DH oracle.

Compute $\tau=\frac{z_{1}}{z_{2}\left(u_{B} \rho_{B} y^{h_{i}}\right)^{s} A}=\rho_{B}^{v} W^{h_{i}}$

Return $\left(i,\left(\tau, h_{i}\right)\right)$

Let $I G$ be the algorithm that generates a random Diffie-Hellman tuple $(\mathbb{G}, g, U, V)$ and $a c c_{\mathcal{B}}$ be the accepting probability of $\mathcal{B} .^{5}$ Then we have that:

$$
a c c_{\mathcal{B}} \geq \frac{\epsilon}{n}-\operatorname{Pr}[\text { bad }=\text { true }]
$$

The probability that bad = true is the probability that the adversary has guessed the "right" $r$ for a corrupted party $I D$ before corrupting it, in one of the $H_{1}$ oracle queries beforehand. Since $r$ is uniformly distributed the probability of guessing it is $1 / q$, and since the adversary makes at most $Q$ queries to $H_{1}$ and corrupts at most $Q_{c}$ parties (and $q>2^{\ell}$ ) we have that

$$
a c c_{\mathcal{B}} \geq \frac{\epsilon}{n}-\frac{Q_{c}(Q)}{2^{\ell}}
$$

which is still non-negligible, since $\epsilon$ is non-negligible.

Once we have described the algorithm $\mathcal{B}$ we can now show how to build a solver algorithm $S$ that can exploit $F_{\mathcal{B}}$, the forking algorithm associated with the above $\mathcal{B}$.

The algorithm $S$ plays the role of a CDH solver under the Strong-DH Assumption. It receives in input a $\mathrm{CDH}$ tuple $(\mathbb{G}, g, U, V)$ where $U=g^{u}, V=g^{v}$ and $u, v$ are random exponents in $\mathbb{Z}_{q} . S$ is also given access to a decision oracle $\operatorname{DH}(U, \cdot, \cdot)$ that on input $(\hat{V}, \hat{W})$ answers "yes" if $(U, \hat{V}, \hat{W})$ is a valid DH tuple.

$$
\begin{aligned}
& \text { Algorithm } S^{\mathrm{DH}(U, \cdot, \cdot)}(\mathbb{G}, g, U, V) \\
& \quad\left(b, \tau, \tau^{\prime}\right) \stackrel{\$}{\leftarrow} F_{\mathcal{B}}^{\mathrm{DH}(U, \cdot, \cdot)}(\mathbb{G}, g, U, V) \\
& \text { If } b=0 \text { then return } 0 \text { and halt } \\
& \text { Parse } \sigma \text { as }(\tau, h) \text { and } \sigma^{\prime} \text { as }\left(\tau^{\prime}, h^{\prime}\right)
\end{aligned}
$$

\footnotetext{
${ }^{5}$ We say that $\mathcal{B}$ accepts if it outputs $(J, \sigma)$ such that $J \geq 1$.
} 


\section{Return $W=\left(\tau / \tau^{\prime}\right)^{\left(h-h^{\prime}\right)^{-1}}$}

If the forking algorithm $F_{\mathcal{B}}$ has success, this means that there exist random coins $\rho$, an index $J \geq 1$ and $h_{1}, \ldots, h_{Q}, h_{J}^{\prime}, \ldots, h_{Q}^{\prime} \in \mathbb{Z}_{q}$ with $h=h_{J} \neq h_{J}^{\prime}=h^{\prime}$ such that: the first execution of $\mathcal{B}\left(\mathbb{G}, g, U, V, h_{1}, \ldots, h_{Q} ; \rho\right)$ outputs $\tau=\rho_{B}^{v} W^{h}$ where $\overline{H_{1}}\left[B, \rho_{B}\right]=h$; the second execution of $\mathcal{B}\left(\mathbb{G}, g, U, V, h_{1}, \ldots, h_{J-1}, h_{J}^{\prime}, \ldots, h_{Q}^{\prime} ; \rho\right)$ outputs $\tau^{\prime}=\left(\rho_{B^{\prime}}^{\prime}\right)^{v} W^{h^{\prime}}$ where $\overline{H_{1}}\left[B^{\prime}, \rho_{B^{\prime}}^{\prime}\right]=h^{\prime}$. Since the two executions of $\mathcal{B}$ are the same until the response to the $J$-th query to $H_{1}$, then we must have $B=$ $B^{\prime}$ and $\rho_{B}=\rho_{B^{\prime}}^{\prime}$. Thus it is easy to see that $S$ achieves its goal by computing $W=\left(\tau / \tau^{\prime}\right)^{\frac{1}{h-h^{\prime}}}=g^{u v}$.

Finally, by the General Forking Lemma, we have that if $\mathcal{A}$ has non-negligible advantage into breaking the security of IB-KA , then $S$ 's success probability is also non-negligible.

\subsection{Additional Security Properties of IB-KA}

In addition to the notion of session key security, any key-agreement protocol should satisfy other important properties. Below we describe the additional security properties enjoyed by IB-KA .

Forward secrecy We say that a KA protocol has forward secrecy, if after a session is completed and its session key erased, the adversary cannot learn it even if it corrupts the parties involved in that session. In other words, learning the private keys of parties should not jeopardize the security of past completed sessions.

A relaxed notion of forward secrecy (which we call weak) assumes that only past sessions in which the adversary was passive (i.e. did not choose the messages) are not jeopardized.

The following theorem shows that the protocol IB-KA satisfies this notion of weak forward secrecy.

Theorem 4. Let $\mathcal{A}$ be a PPT adversary that is able to break the weak forward secrecy of the IB-KA protocol with advantage $\epsilon$. Let $n$ be the an upper bound to the number of sessions of the protocol run by $\mathcal{A}$ and $Q_{1}$ and $Q_{2}$ be the number of queries made by the adversary to the random oracles $H_{1}, H_{2}$ respectively. Then we can solve the $C D H$ problem with probability at least $\epsilon /\left(n Q_{2}\right)$.

Proof. For sake of contradiction let us suppose there exists a PPT adversary $\mathcal{A}$ that is able to break the weak forward secrecy of the protocol IB-KA with non-negligible advantage $\epsilon$. Then we show how to build a simulator $S$ that uses $\mathcal{A}$ to solve the $\mathrm{CDH}$ problem with probability at least $\epsilon / n Q_{2}$. $S$ receives in input a tuple $(\mathbb{G}, g, U, V)$ where $U=g^{u}, V=g^{v}$ and $u, v$ are random exponents in $\mathbb{Z}_{q}$. The simulator plays the role of the $\mathrm{CDH}$ solver and its goal it to compute the value $W=g^{u v}$.

Setup. $S$ sets up a simulated execution of the protocol, with simulated KGC, users and sessions. First of all $S$ defines the public parameters of the protocol simulating the KGC. So it chooses a random $x \stackrel{\$}{\leftarrow} \mathbb{Z}_{q}$ and sets $y=g^{x}$. Then it provides the adversary with input $(\mathbb{G}, g, y)$ and oracle access to $H_{1}$ and $H_{2}$. Since $H_{1}$ and $H_{2}$ are modeled as random oracles, $S$ can program their output. For each input $\left(I D, r_{I D}\right) S$ chooses a random $e_{I D} \stackrel{\$}{\leftarrow} \mathbb{Z}_{q}$ and sets $H_{1}\left(I D, r_{I D}\right)=e_{I D}$.

Since $S$ knows the master secret key $x$, it can simulate the KGC in full, and give secret keys to all the parties in the network, including answering private key queries from the adversary.

At the beginning of the game $S$ guesses the test session and its peers Alice and Bob.

Simulating protocol sessions. Sessions different from the test session are easily simulated since $S$ knows all the information needed to compute the session keys and answer any query (including session key and state reveal queries) from the adversary. 
Simulating the test session. We now show how to simulate the test session in order to extract $W=g^{u v}$ from the adversary. Since in this game the adversary is assumed to be passive during the test session, the parties (i.e. the simulator in this case) choose the messages exchanged in this session.

Let $\left(A, r_{A}, s_{A}\right),\left(B, r_{B}, s_{B}\right)$ be the identity information and the secret keys of Alice and Bob respectively ( $S$ knows these values). The simulator sets Alice's message as $\left(A, r_{A}, u_{A}=U\right)$ while the one from Bob is $\left(B, r_{B}, u_{B}=V\right) . S$ is implicitly setting $t_{A}=u, t_{B}=v$. In this case the correct session key is $Z=H_{2}\left(g^{\left(s_{A}+u\right)\left(s_{B}+v\right)}, g^{u v}\right)$. Since $H_{2}$ is modeled as a random oracle, if $\mathcal{A}$ has success into distinguishing $Z$ from a random value, it must have queried $H_{2}$ on the correct input $\left(z_{1}=g^{\left(s_{A}+u\right)\left(s_{B}+v\right)}, z_{2}=g^{u v}\right)$. Thus $S$ can choose a random value among all the queries that it received from the adversary. Since the number of queries $Q_{2}$ is polynomially bounded, $S$ can find the correct $z_{2}=W$ with non-negligible probability $\epsilon / n Q_{2}$. This completes the proof of this case ${ }^{6}$.

Resistance to reflection attacks A reflection attack occurs when an adversary can compromise a session in which the two parties have the same identity (and the same private key). Though, at first glance, this seems to be only of theoretical interest, there are real-life situations in which this scenario occurs. For example consider the case when Alice is at her office and wants to establish a secure connection with her PC at home, therefore running a session between two computers with the same identity and private key.

Here we extend the proof of security given in Section 4 to support reflection attacks. We observe that in the case when the test session has a matching session the proof remains valid even if the test session is between Bob and himself.

On the other hand, when there is no matching session we have to show a little modification of the proof. In fact the current proof actually does not work when the adversary sends a message with the same value $r_{B}$ provided by the KGC (for which the simulator knows the discrete logarithm $k_{B}$, but cannot compute the corresponding $s_{B}$ ). The issue is that the knowledge of $s_{B}$ would be needed to extract the solution of the CDH problem.

We point out that a reflection attack using a value $\rho_{B} \neq r_{B}$ is captured by the current proof. Moreover it is reasonable to assume that a honest party refuses connections from itself that use a "wrong" key.

However it is possible to adapt the proof in this specific case. In particular we can show that a successful run of the adversary enables the simulator to compute $g^{u^{2}}$ instead of $g^{u v}$. As showed in [27] by Maurer and Wolf, such an algorithm can be easily turned into a solver for CDH. For lack of space this is deferred to the full version of the paper.

In this section we show how to adapt the proof in this specific case. In particular, we show that a successful run of the adversary enables the simulator to compute $g^{u^{2}}$ instead of $g^{u v}$. As showed in [27] by Maurer and Wolf, such an algorithm can be easily turned into a solver for CDH.

Let us consider the following modification of the proof given in Section 4. If in the test session the adversary sends a message from Bob to Bob of type $\left\langle B, r_{B}, u_{B}=g^{t_{B}}\right\rangle$ then the simulator picks a random $e \stackrel{\$}{\leftarrow} \mathbb{Z}_{q}$ and replies with message $\left\langle B, r_{B}, u_{B}^{\prime}=U^{e}\right\rangle$. Let $h^{*}$ be the random oracle response to $H_{1}\left(B, r_{B}\right)$. We observe that in this case the correct session key is the hash $Z=H_{2}\left(z_{1}, z_{2}\right)$ where $z_{1}=g^{\left(k_{B}+u h^{*}+u e\right)\left(k_{B}+u h^{*}+t_{B}\right)}$ and $z_{2}=g^{u e t_{B}}$. If the adversary has success into distinguishing $Z$

\footnotetext{
${ }^{6}$ We could give the simulator access to the Strong-DH oracle $\mathrm{DH}$, and then $S$ could use it to "test" all queries to $H_{2}$ to find the correct $W$. The reduction would be tighter (removing the factor of $Q_{2}^{-1}$ from the success probability) but would require the Strong-DH Assumption also in this case.
} 
from a random value it must necessarily query the correct pair $\left(z_{1}, z_{2}\right)$ to the random oracle $H_{2}$. This means that $S$ can efficiently find the pair $\left(z_{1}, z_{2}\right)$ in the table $\overline{H_{2}}$ using the Strong-DH oracle. Once it has recovered these values, it can compute:

$$
g^{u^{2}}=\left(\frac{z_{1}}{g^{k_{B}^{2}} U^{2 k_{B} h^{*}} U^{e k_{B}} u_{B}^{k_{B}} z_{2} z_{2}^{h^{*} / e}}\right)^{\frac{1}{h^{*}\left(h^{*}+e\right)}} .
$$

Resistance to Key Compromise Impersonation Suppose that the adversary learns Alice's private key. Then, it is trivial to see that this knowledge enables the adversary to impersonate Alice to other parties. A key compromise impersonation (KCI) attack can be carried out when the knowledge of Alice's private key allows the adversary to impersonate another party to Alice.

To see that the protocol IB-KA is resistant to KCI attacks it suffices to observe that in the proof of security, when the adversary tries to impersonate Bob to Alice, we are able to output Alice's private key whenever it is asked by the adversary. It means that the proof continues to be valid even in this case.

Ephemeral Key Compromise Impersonation A recent paper by Cheng and Ma [14] shows that our protocol is susceptible to an ephemeral key compromise attack. Roughly speaking this attack considers the case when the adversary can make state-reveal queries (in order to learn the ephemeral key of a user) even in the test session. Though the paper is correct, we point out that this kind of attack is not part of the standard Canetti-Krawczyk security model that is considered in this paper.

\section{A protocol secure under $\mathrm{CDH}$}

The protocol IB-KA given in section Section 3 is proven secure under the Strong-DH Assumption. In this section we show how to modify that protocol so that its security can be based directly on $\mathrm{CDH}$. The cost is a few more exchanged elements and a few more exponentiations.

The basic idea is to use the Twin Diffie-Hellman (2DH) Assumption introduced by Cash et al. in [11]. Informally $2 \mathrm{DH}$ states that an adversary which is given in input random $U_{1}, U_{2}, V \in \mathbb{G}$, should not be able to compute a pair $\left(W_{1}, W_{2}\right)$ such that $W_{1}$ and $W_{2}$ are the DH of $U_{1}, V$ and $U_{2}, V$ respectively. It is easy to see that this assumption is equivalent to the well known CDH. The valuable contribution of [11] was to show that its "strong" version is equivalent to CDH too.

Informally the Strong-2DH assumption says that $2 \mathrm{DH}$ holds even in the presence of an oracle $2 \mathrm{DH}\left(U_{1}, U_{2}, \cdot, \cdot, \cdot\right)$ that solves its decisional version for fixed $U_{1}, U_{2}$.

Therefore we modify the IB-KA protocol in such a way it can be proven secure under the Strong2DH Assumption. Then, since Cash et al. proved in [11] that Strong-2DH and CDH are equivalent, we obtain a protocol secure under $\mathrm{CDH}$.

In order to modify the protocol we apply the idea of "twinning" some elements so that the construction can be proven under the Strong-2DH assumption. The new protocol is almost the same as IB-KA except for the following:

- the master public key consists of two group elements $y_{1}, y_{2}$. This means that each user $I D$ owns a secret key $\left(r_{I D}^{1}, s_{I D}^{1}, r_{I D}^{2}, s_{I D}^{2}\right)$ which are two Schnorr's signatures of its identity corresponding to public keys $y_{1}, y_{2}$ respectively. 
- each user $I D$ generates two elements $u_{I D}^{1}=g^{t_{I D}^{1}}, u_{I D}^{2}=g^{t_{I D}^{2}}$ and sends $\left\langle r_{I D}^{1}, r_{I D}^{2}, u_{I D}^{1}, u_{I D}^{2}\right\rangle$.

- the session key of a session between users with identities $A$ and $B$ is

$$
K=H\left(z_{11}, z_{12}, z_{21}, z_{22}, \omega_{11}, \omega_{12}, \omega_{21}, \omega_{22}\right)
$$

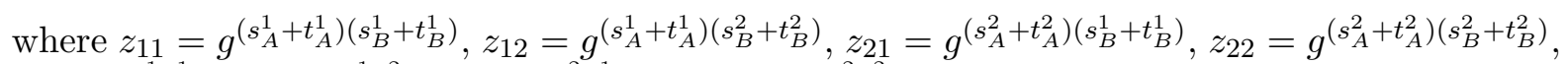
$\omega_{11}=g^{t_{A}^{1}} t_{B}^{1}, \omega_{12}=g^{t_{A}^{1}} t_{B}^{2}, \omega_{21}=g^{t_{A}^{2} t_{B}^{1}}$ and $\omega_{22}=g^{t_{A}^{2} t_{B}^{2}}$.

It is also possible to instantiate a simpler version of this protocol in which the public key is only $y$ as in IB-KA. This is slightly more efficient since a user has to send one less element. This variant can also be proven secure under the $\mathrm{CDH}$ provided that the adversary is not allowed to issue state-reveal queries.

The proof of security of this protocol will appear in an extended version of this paper.

\section{Comparisons with other IB-KA Protocols}

In this section we compare IB-KA with other id-based KA protocols from the literature. In particular, we consider the protocol by Chen and Kudla [13] (SCK-2) (which is a modification of Smart's [35]) and two protocols proposed very recently by Boyd et al. [7] (BCNP1, BCNP2).

For our efficiency comparisons we consider a security parameter of 128 and implementations of SCK-2, BCNP1 and BCNP2 with Type 3 pairings ${ }^{7}$, which are the most efficient pairings for this kind of security level (higher than 80). Our protocol is assumed to be implemented in an elliptic curves group $\mathbb{G}$ with the same security parameter. In this scenario elements of $\mathbb{G}$ and $\mathbb{G}_{1}$ need 256 bit to be represented, while 512 bits are needed for $\mathbb{G}_{2}$ elements and 3072 bits for an element of $\mathbb{G}_{T}$.

We estimate the computational cost of all the protocols using the costs per operation for Type 3 pairings given by Chen et al. in [12]. The bandwidth cost is expressed as the amount of data in bits sent by each party to complete a session of the protocol ${ }^{8}$.

According to the work of Chen et al. [12] SCK-2 is the most efficient protocol with a proof of security in the CK model for all types of pairings. It is proved secure using random oracles under the Bilinear Diffie-Hellman Assumption and requires one round of communication with only one group element sent by each party. To be precise, we point out that the protocol of Boyd et al. (BMP) [8] would appear computationally more efficient than SCK-2, but unfortunately it works only in type 1 and type 4 pairings and is proven secure only in symmetric pairings. BCNP1 and BCNP2 are generic constructions based on any CCA-secure IB-KEM. When implemented (as suggested by the authors of [7]) using one of the IB-KEMs by Kiltz [23], Kiltz-Galindo [24] or Gentry [20] they lead to a two-pass single-round protocol with (CK) security in the standard model. BCNP2 provides weak FS and resistance to KCI attacks, while BCNP1 satisfies only the former property.

The results are summarized in Table 1 assuming protocols BCNP1 and BCNP2 to be implemented with Kiltz's IB-KEM (the most efficient for this application according to the work of Boyd et al. [7]). We defer to the original papers of SCK-2 [13] and BCNP1, BCNP2 [7] for more details about these costs. As described in the table, our protocol has a reasonable bandwidth requirement and achieves the best computational efficiency among the other id-based KA protocols.

\footnotetext{
7 This classification of pairing groups into several types is provided by Galbraith et al. in [18].

${ }^{8}$ We do not consider the identity string sent with the messages as it can be implicit and, in any way, appears in all the protocols.
} 


\begin{tabular}{|c|c|c|c|c|c|}
\hline & weak & \multirow{2}{*}{ KCI } & Standard & \multicolumn{2}{|c|}{ Efficiency } \\
\cline { 5 - 6 } & FS & & model & Bandwidth & Cost per party \\
\hline BCNP1 & $\boldsymbol{X}$ & $\checkmark$ & $\checkmark$ & 768 & 56 \\
BCNP2 & $\checkmark$ & $\checkmark$ & $\checkmark$ & 1024 & 59 \\
SCK-2 & $\checkmark$ & $\checkmark$ & $\boldsymbol{x}$ & 256 & 43 \\
IB-KA & $\boldsymbol{\checkmark}$ & $\boldsymbol{\checkmark}$ & $\boldsymbol{x}$ & 512 & 6 \\
\hline
\end{tabular}

Table 1. Comparisons between IB-KA protocols.

Comparison with PKI-BASED PRotocols. We also compare our protocol to MQV [26], and its provably secure version HMQV [25], which is the most efficient protocol in the public-key setting. When comparing our protocol to a PKI-based scheme, like MQV, we must also consider the additional cost of sending and verifying certificates.

We measure the computation costs of the protocols in terms of the number of exponentiations in the underlying group needed to compute the session key. If the exponentiations is done with an exponent that is half the length of the group size, then obviously we count it as $1 / 2$ exponentiation. Also if an exponentiation is done over a fixed basis, we apply precomputation schemes to speed up the computation, e.g. [19].

Our protocol requires each party to send a single message consisting of two group elements. To compute the session key, the parties perform 2 full exponentiations over variable basis, and one half exponentiation over a fixed basis ${ }^{9}$. For our security parameter, following [19], the latter half exponentiation can be computed with less than 20 group multiplications, with a precomputation table of moderate size.

In MQV, each party sends a single message consisiting of one group element, and performs 1.5 exponentiations to compute the session key. Moreover, in HMQV certificates are sent and verified. Here we distinguish two cases: the certificate is based either on an RSA signature, or on a discretelog signature, e.g. Schnorr's.

In the RSA case, a short exponent e.g. $e=2^{16}+1$, is typically used, and the verification cost is basically equivalent to the cost of the half exponentiation with precomputation in our protocol above. Therefore in this case, MQV is faster, but by a mere half exponentiation. The price to pay however is a massive increase in bandwidth to send the RSA signature (i.e. 3072 bits), and the introduction of the RSA Assumption in order to prove security of the entire scheme.

If we use a Schnorr signature for the certificate, then MQV require sending two more group elements, and therefore its bandwidth requirement is already worse than our protocol (by one group element). The parties then must compute one full and one half exponentiation, both with fixed basis $^{10}$ to verify the certificate. This extra computational cost can be compared to an additional half exponentiation, making the computation requirement of MQV with Schnorr certificates equivalent to that of our protocol.

In conclusion, when comparing our protocol with MQV with certificates we find that our protocol: (i) has comparable computational cost; (ii) has better bandiwdth (by far in the case of RSA certificates) and (iii) simplifies protocol implementation by removing entirely the need to manage certificates and to interact with a $\mathrm{PKI}^{11}$.

\footnotetext{
${ }^{9}$ Indeed since the input to the hash function $H_{1}$ is randomized, we can set its output length to be half of the length of the group size.

10 Though different basis, which means that in order to apply precomputation techniques, the parties need to maintain two tables.

${ }^{11}$ In the above, we did not account for the cost of verifying group membership for the elements sent by the parties, which is necessary both in the case of MQV and our protocol, and is the same in both protocols.
} 


\section{References}

1. M. Abdalla, M. Bellare and P. Rogaway. The oracle Diffie-Hellman assumptions and an analysis of DHIES. In proceedings of CT-RSA 2001, LNCS vol. 2020, pp. 143-158.

2. M. Bellare and G. Neven. New Multi-Signature Schemes and a General Forking Lemma. In proceedings of the 13th Conference on Computer and Communications Security - ACM CCS 2006, ACM Press, 2006.

3. M. Bellare and A. Palacio. The Knowledge-of-Exponent Assumptions and 3-round Zero-Knowledge Protocols. Advances in Cryptology - CRYPTO 2004, LNCS vol. 3152.

4. Dan Boneh, Xavier Boyen. Short Signatures without Random Oracles. Advances in Cryptology - Eurocrypt 2004, LNCS vol. 3027.

5. Dan Boneh, Matthew K. Franklin. Identity-Based Encryption from the Weil Pairing. SIAM J. Comput. 32(3): 586-615 (2003) (Also in CRYPTO 2001.)

6. Colin Boyd, Kim-Kwang Raymond Choo. Security of Two-Party Identity-Based Key Agreement. Mycrypt 2005: 229-243

7. Colin Boyd, Yvonne Cliff, Juan Gonzalez Nieto, Kenneth G. Paterson. Efficient One-Round Key Exchange in the Standard Model. In proceedings of ACISP 2008, LNCS vol. 5107, pp. 69-83

8. Colin Boyd, Wenbo Mao, Kenneth G. Paterson. Key Agreement Using Statically Keyed Authenticators. In proceedings of ACNS 2004, LNCS vol. 3089, pp. 248-262

9. R. Canetti, H. Krawczyk. Analysis of Key-Exchange Protocols and Their Use for Building Secure Channels. Advances in cryptology - EUROCRYPT 2001, LNCS vol. 2045, pp. 453-474

10. R. Canetti, H. Krawczyk. Universally Composable Notions of Key Exchange and Secure Channels. Advances in cryptology - EUROCRYPT 2002, LNCS vol. 2332, pp. 337-351

11. D. Cash, E. Kiltz and V. Shoup. The Twin Diffie-Hellman Problem and Applications Advances in cryptology EUROCRYPT 2008, LNCS vol. 4965, pp. 127-145.

12. L. Chen, Z. Cheng, Nigel P. Smart. Identity-based key agreement protocols from pairings. Int. J. Inf. Sec. 6(4): 213-241 (2007)

13. L. Chen and C. Kudla. Identity Based Authenticated Key Agreement Protocols from Pairings In 16th IEEE Computer Security Foundations Workshop - CSFW 2003, pages 219-233. IEEE Computer Society Press, 2003.

14. Q. Cheng and C. Ma. Ephemeral Key Compromise Attack on the IB-KA protocol. Cryptology Eprint Archive, Report 2009/568. http://eprint.iacr.org/2009/568.

15. I. Damgård. Towards Practical Public Key Systems Secure Against Chosen Ciphertext Attacks. Advances in Cryptology - CRYPTO'91, LNCS vol. 576.

16. W. Diffie and M. Hellman. New Directions in Cryptography. IEEE Transactions on Information Theory, 1976, vol. 22 , n. 6 , pp. $644-654$

17. A. Fiat and A. Shamir How to Prove Yourself: Practical Solutions of Identification and Signature Problems. Advances in cryptology - CRYPTO 1986, LNCS vol. 263, pp. 186-194

18. S.D. Galbraith, K.G. Paterson and N.P. Smart. Pairings for Cryptographers. Cryptology ePrint Archive, Report 2006/165, 2006. http://eprint.iacr.org.

19. C.H. Lim and P.J. Lee More Flexible Exponentiation with Precomputation In Crypto'94, pp.95-107, LNCS no. 839 .

20. C. Gentry. Practical Identity-Based Encryption Without Random Oracles. Advances in cryptology - proceedings of EUROCRYPT 2006, 2006, LNCS vol. 4004, pp. 494-510

21. Gunther, C.G. An Identity-Based Key-Exchange Protocol. Advances in cryptology-proceedings of EUROCRYPT 1989, 1989, LNCS, vol. 434, pp. 29-37.

22. S. Hada and T. Tanaka. On the Existence of 3-round Zero-Knowledge Protocols. Advances in Cryptology CRYPTO 1998, LNCS vol. 1462

23. E. Kiltz. Direct Chosen-Ciphertext Secure Identity-Based Encryption in the Standard Model with short Ciphertexts. Cryptology Eprint Archive, Report 2006/122. http://eprint.iacr.org/2006/122.

24. E. Kiltz and D. Galindo. Direct Chosen-Ciphertext Secure Identity-Based Key Encapsulation Without Random Oracles. Cryptology Eprint Archive, Report 2006/034. http://eprint.iacr.org/2006/034.

25. Hugo Krawczyk. HMQV: A High-Performance Secure Diffie-Hellman Protocol. Advances in cryptology CRYPTO 2005, LNCS vol. 3621, pp. 546-566

26. L. Law, A. Menezes, M. Qu, J. Solinas, and S. Vanstone. An efficient Protocol for Authenticated Key Agreement. Designs, Codes and Cryptography, 28, 119-134, 2003.

27. U. Maurer and S. Wolf. Diffie-Hellman oracles. Advances in cryptology - CRYPTO 1996, LNCS vol. 1109, pp. 268-282 
28. E. Okamoto. Key Distribution Systems Based on Identification Information. In Advances in Cryptology, Crypto 1987, pp. 194-202. LNCS Vol. 293/1988.

29. E. Okamoto and K. Tanaka. Key Distribution System Based on Identification. Information. IEEE Journal on Selected Areas in Communications, 7(4):481-485, May 1989.

30. D. Pointcheval and J. Stern. Security Arguments for Digital Signatures and Blind Signatures. Journal of Cryptology, 13(3):361-396 (2000).

31. S. Saeednia. Improvement of Gunther's identity-based key exchange protocol. Electonics Letters vol. 36, Issue 18, 31 Aug 2000, pp. 1535 - 1536

32. R. Sakai, K. Ohgishi and M. Kasahara. Cryptosystems based on pairing. In Symposium on Cryptography and Information Security, Okinawa, Japan, 2000.

33. Adi Shamir Identity-Based Cryptosystems and Signature Schemes Advances in Cryptology - Proceedings of CRYPTO '84, 1985, pp. 47-53

34. C.P. Schnorr. Efficient identification and signatures for smart cards. Advances in Cryptology - CRYPTO '89, 1989, LNCS vol. 435, pp. 239-252

35. N. P. Smart. An identity-based authenticated key-agreement protocol based on the Weil pairing. Electronics letters, 2002, vol. 38, pp.630-632.

36. D.K. Smetters, G. Durfee. Domain-based Administration of Identity-Based Cryptosystems for Secure E-Mail and IPSEC. In: SSYM 2003: Proceedings of the 12th Conference on USENIX Security Symposium, p. 15. USENIX Association (2003)

37. Y. Wang. Efficient Identity-Based and Authenticated Key Agreement Protocol. Cryptology ePrint Archive, Report 2005/108, 2005. http://eprint.iacr.org/2005/108/. 